\title{
A SYSTEM OF NONLINEAR SET-VALUED IMPLICIT VARIATIONAL INCLUSIONS IN REAL BANACH SPACES
}

\author{
Chuanzhi Bai And Qing Yang
}

\begin{abstract}
In this paper, we introduce and study a system of nonlinear set-valued implicit variational inclusions (SNSIVI) with relaxed cocoercive mappings in real Banach spaces. By using resolvent operator technique for $M$-accretive mapping, we construct a new class of iterative algorithms for solving this class of system of set-valued implicit variational inclusions. The convergence of iterative algorithms is proved in $q$-uniformly smooth Banach spaces. Our results generalize and improve the corresponding results of recent works.
\end{abstract}

\section{Introduction}

In [4], Fang and Huang first introduced the concept of $H$-accretive operators in Banach spaces. Obviously, the class of $H$-accretive operators provides a unifying frameworks for classes of maximal monotone operators, $m$-accretive operators and $H$-monotone operator [5]. Very recently, Chang, Joseph Lee, and Chan [2] introduced and studied a class of generalized system for relaxed cocoercive variational inequalities in Hilbert spaces which extended and improved the main results in $[6,8,9]$.

Inspired and motivated by the results in $[1,2,4,6]$, the purpose of this paper is to introduce and study a system of nonlinear implicit variational inclusions in Banach spaces. By using the resolvent operator technique for the $M$-accretive mapping, we develop a new class of iterative algorithms to solve a class of relaxed cocoercive set-valued implicit variational inclusions associated with $M$ accretive mappings in $q$-uniformly smooth Banach spaces. Our results improve and extend the corresponding results of Chang, Joseph Lee, and Chan [2].

Let $E$ be an arbitrary real Banach space and let $J_{q}(q>1)$ denote the generalized duality mapping from $E$ into $2^{E^{*}}$ given by

$$
J_{q}(x)=\left\{f \in E^{*}:\langle x, f\rangle=\|x\|^{q},\|f\|=\|x\|^{q-1}\right\},
$$

Received July 25, 2007.

2000 Mathematics Subject Classification. Primary 49J40, 47H04.

Key words and phrases. system of nonlinear set-valued implicit variational inclusion, resolvent operator, $M$-accretive mapping, iterative algorithm, convergence. 
where $E^{*}$ denotes the dual space of $E$ and $\langle\cdot, \cdot\rangle$ denotes the generalized duality pairing. In particular, $J_{2}$ is called the normalized duality mapping and it is usually denoted by $J$.

The modulus of smoothness of $E$ is the function $\rho_{E}:[0, \infty) \rightarrow[0, \infty)$ defined by

$$
\rho_{E}(t)=\sup \left\{\frac{1}{2}(\|x+y\|+\|x-y\|)-1:\|x\| \leq 1,\|y\| \leq t\right\} .
$$

A Banach space $E$ is called uniformly smooth if $\lim _{t \rightarrow 0} \frac{\rho_{E}(t)}{t}=0 . E$ is called $q$-uniformly smooth if there exists a constant $c>0$ such that

$$
\rho_{E}(t) \leq c t^{q}, \quad q>1 .
$$

It is known that, $J_{q}$ is single-valued if $E$ is uniformly smooth.

Definition 1.1. Let $E$ be a real uniformly smooth Banach space, and $M$ : $E \rightarrow E$ be a single-valued operator. Then $M$ is said to be:

(i) accretive, if

$$
\left\langle M x-M y, J_{q}(x-y)\right\rangle \geq 0, \quad \forall x, y \in E ;
$$

(ii) strictly accretive, if $M$ is accretive and

$$
\left\langle M x-M y, J_{q}(x-y)\right\rangle=0 \text { if and only if } x=y ;
$$

(iii) strongly accretive, if there exists a constant $r>0$ such that

$$
\left\langle M x-M y, J_{q}(x-y)\right\rangle \geq r\|x-y\|^{q}, \quad \forall x, y \in E .
$$

Let $C(E)$ denote the family of all nonempty compact subsets of $E$. We introduce a new definition as follows.

Definition 1.2. Let $E$ be a real uniformly smooth Banach space, $T: E \times E \rightarrow$ $E$ and $g: E \rightarrow E$ be two single-valued mappings, and $V: E \rightarrow C(E)$ be a set-valued mapping.

(i) $T$ is said to be relaxed $(\omega, r)$-cocoercive with respect to $(V, g)$ in the first variable, if there exist constants $w, r>0$ such that for all $x_{1}, x_{2} \in E$, $u_{1} \in V\left(x_{1}\right)$ and $u_{2} \in V\left(x_{2}\right)$

$$
\begin{aligned}
& \left\langle T\left(u_{1}, v_{1}\right)-T\left(u_{2}, v_{2}\right), J_{q}\left(g\left(x_{1}\right)-g\left(x_{2}\right)\right)\right\rangle \\
\geq & (-\omega)\left\|T\left(u_{1}, v_{1}\right)-T\left(u_{2}, v_{2}\right)\right\|^{q}+r\left\|x_{1}-x_{2}\right\|^{q}, \quad \forall v_{1}, v_{2} \in E .
\end{aligned}
$$

(ii) $T$ is said to be $\mu$-Lipschitz continuous, if there exists a constant $\mu>0$ such that for all $u_{1}, u_{2} \in E$

$$
\left\|T\left(u_{1}, v_{1}\right)-T\left(u_{2}, v_{2}\right)\right\| \leq \mu\left\|g\left(u_{1}\right)-g\left(u_{2}\right)\right\|, \quad \forall v_{1}, v_{2} \in E .
$$

Remark 1.1. If $E=H$ is a Hilbert space, $g=I$ (the identity map on $E$ ) and $V: E \rightarrow E$ is a single-valued with $V=I$, then (i) of Definition 2.2 reduces to the definition of relaxed $(\gamma, r)$-cocoercive mappings in [2]. 
Definition 1.3. The set-valued operator $W: E \rightarrow C(E)$ is said to be $\widetilde{H}$ Lipschitz continuous, if there exists a constant $\xi>0$ such that

$$
\widetilde{H}(W(u), W(v)) \leq \xi\|u-v\|, \quad \forall u, v \in E,
$$

where $\widetilde{H}(\cdot, \cdot)$ is the Hausdorff metric on $C(E)$.

Definition 1.4. Let $M: E \rightarrow E$ be a single-valued operator and $A: E \rightarrow 2^{E}$ be a multivalued operators. $A$ is said to be $M$-accretive if $A$ is accretive and $(M+\lambda A)(E)=E$ holds for all $\lambda>0$.

Remark 1.2. From [4], it is easily established that if $M=I$ (the identity map on $E)$, then the definition of $I$-accretive operators is that of $m$-accretive operators. Conversely, the Example 2.1 in [4] shows that an $m$-accretive operator need not be $M$-accretive for some $M$.

Remark 1.3. It is well known that, if $E=H$ is a Hilbert space, then $A: D(A) \subset$ $H \rightarrow 2^{H}$ is an $m$-accretive mapping if and only if $A: D(A) \subset H \rightarrow 2^{H}$ is a maximal monotone mapping (see, for example, [3]).

Let $M, g: E \rightarrow E, T: E \times E \rightarrow E$ be three single-valued operators, $V, W:$ $E \rightarrow C(E)$ be two set-valued mappings and $A: E \rightarrow 2^{E}$ be a $M$-accretive operator. We consider a system of nonlinear implicit set-valued variational inclusion (abbreviated as SNISVI) problem as follows: to find $x^{*}, y^{*} \in E$, $u^{*} \in V\left(x^{*}\right), v^{*} \in W\left(y^{*}\right)$ such that

$$
\begin{array}{ll}
\theta \in \rho T\left(v^{*}, u^{*}\right)+M\left(x^{*}\right)-g\left(y^{*}\right)+A\left(x^{*}\right) & \text { for } \rho>0, \\
\theta \in \eta T\left(u^{*}, v^{*}\right)+M\left(y^{*}\right)-g\left(x^{*}\right)+A\left(y^{*}\right) & \text { for } \eta>0,
\end{array}
$$

where $\theta$ is a zero element in $E$. Below are some special cases of the SNISVI problem (1) and (2).

(i) If $V, W: E \rightarrow E$ are two single-valued mappings, then the SNISVI problem (1) and (2) can be replaced by finding $x^{*}, y^{*} \in E$ such that

$$
\begin{array}{ll}
\theta \in \rho T\left(W\left(y^{*}\right), V\left(x^{*}\right)\right)+M\left(x^{*}\right)-g\left(y^{*}\right)+A\left(x^{*}\right) & \text { for } \rho>0, \\
\theta \in \eta T\left(V\left(x^{*}\right), W\left(y^{*}\right)\right)+M\left(y^{*}\right)-g\left(x^{*}\right)+A\left(y^{*}\right) & \text { for } \eta>0 .
\end{array}
$$

(ii) If $V=W=I$, then the system of nonlinear implicit variational inclusion (abbreviated as SNIVI) problem (3) and (4) is equivalent to finding $x^{*}, y^{*} \in E$ such that

$$
\begin{array}{ll}
\theta \in \rho T\left(y^{*}, x^{*}\right)+M\left(x^{*}\right)-g\left(y^{*}\right)+A\left(x^{*}\right) & \text { for } \rho>0, \\
\theta \in \eta T\left(x^{*}, y^{*}\right)+M\left(y^{*}\right)-g\left(x^{*}\right)+A\left(y^{*}\right) & \text { for } \eta>0 .
\end{array}
$$

(iii) If $E=H$ is a Hilbert space, $M=I$ and $A=\partial \phi$, where $\phi: H \rightarrow$ $R \cup\{+\infty\}$ is a proper convex lower semicontinuous function on $H$ and $\partial \phi$ denotes the subdifferential of function $\phi$, then the SNIVI problem (5) and (6) is equivalent to finding $x^{*}, y^{*} \in H$ such that

(7) $\left\langle\rho T\left(y^{*}, x^{*}\right)+x^{*}-g\left(y^{*}\right), x-x^{*}\right\rangle \geq \phi\left(x^{*}\right)-\phi(x), \quad \forall x \in H$ and $\rho>0$, 
(8) $\left\langle\eta T\left(x^{*}, y^{*}\right)+y^{*}-g\left(x^{*}\right), x-y^{*}\right\rangle \geq \phi\left(y^{*}\right)-\phi(x), \quad \forall x \in H$ and $\eta>0$.

(iv) If $E=H$ is a Hilbert space, $M=g=I$ and $\phi$ is the indicator function of a closed convex subset $K$ in $H$, that is,

$$
\phi(u)=I_{K}(u)= \begin{cases}0, & u \in K, \\ +\infty, & \text { other, }\end{cases}
$$

then the SNIVI problem (7) and (8) is equivalent to finding $x^{*}, y^{*} \in K$ such that

$$
\begin{aligned}
& \left\langle\rho T\left(y^{*}, x^{*}\right)+x^{*}-y^{*}, x-x^{*}\right\rangle \geq 0, \quad \forall x \in H \text { and } \rho>0, \\
& \left\langle\eta T\left(x^{*}, y^{*}\right)+y^{*}-x^{*}, x-y^{*}\right\rangle \geq 0, \quad \forall x \in H \text { and } \eta>0,
\end{aligned}
$$

which is called a system of nonlinear variational inequality (SNVI) introduced and studied by Chang, Joseph Lee, and Chan [2].

Let $M: E \rightarrow E$ be a strictly accretive operator and $A: E \rightarrow 2^{E}$ is a $M$ accretive operator. Fang and Huang [4] defined the resolvent operator $J_{M}^{A, \lambda}$ : $E \rightarrow E$ associated with $A, M$ and $\lambda$ as follows:

$$
J_{A, \lambda}^{M}(u)=(M+\lambda A)^{-1}(u), \quad \forall u \in E .
$$

From the proof of Theorem 2.3 in [4], it is easy to obtain the following result.

Lemma 1.5. Let $M: E \rightarrow E$ be a strongly accretive operator with constant $k>0$ and $A: E \rightarrow 2^{E}$ is a $M$-accretive operator. Then the resolvent operator $J_{A, \lambda}^{M}: E \rightarrow E$ is Lipschitz continuous with constant $\frac{1}{k}$, i.e.,

$$
\left\|J_{A, \lambda}^{M}(x)-J_{A, \lambda}^{M}(y)\right\| \leq \frac{1}{k}\|x-y\|, \quad \forall x, y \in E .
$$

\section{Main results}

Throughout this section, we always let $E$ a real $q$-uniformly smooth Banach space. In [10], Xu proved the following result.

Lemma 2.1. If $E$ is a real $q$-uniformly smooth Banach space, then there exists a constant $c_{q} \geq 1$ such that

$$
\|x+y\|^{q} \leq\|x\|^{q}+q\left\langle y, J_{q}(x)\right\rangle+c_{q}\|y\|^{q}, \quad \forall x, y \in E .
$$

For convenience, let $J_{A, 1}^{M}:=J_{A}^{M}$. To obtain the approximate solution of the SNISVI problem (1) and (2), we first give a characterization the solution of problem (1) and (2) by using the resolvent operator $J_{A}^{M}$.

Lemma 2.2. Let $A: E \rightarrow 2^{E}$ is a $M$-accretive. Then $\left(x^{*}, y^{*}, u^{*}, v^{*}\right)$ is a solution to the SNISVI problem (1) and (2) if and only if $\left(x^{*}, y^{*}, u^{*}, v^{*}\right)$ satisfies

$$
\begin{array}{ll}
x^{*}=J_{A}^{M}\left[g\left(y^{*}\right)-\rho T\left(v^{*}, u^{*}\right)\right] & \text { for } \rho>0, \\
y^{*}=J_{A}^{M}\left[g\left(x^{*}\right)-\eta T\left(u^{*}, v^{*}\right)\right] & \text { for } \eta>0 .
\end{array}
$$

Proof. By using the definition of $J_{A}^{M}$, we can prove this lemma immediately. 
Based on Lemma 2.2, we construct the following iterative algorithm for solving problem (1) and (2).

Algorithm 2.1. For given $x_{0}, y_{0} \in E, u_{0} \in V\left(x_{0}\right), v_{0} \in W\left(y_{0}\right)$, compute the sequences $\left\{x_{n}\right\},\left\{y_{n}\right\},\left\{u_{n}\right\}$ and $\left\{v_{n}\right\}$ from the iterative schemes

$$
\begin{gathered}
u_{n} \in V\left(x_{n}\right):\left\|u_{n}-u_{n+1}\right\| \leq \widetilde{H}\left(V\left(x_{n}\right), V\left(x_{n+1}\right)\right), \\
v_{n} \in W\left(y_{n}\right):\left\|v_{n}-v_{n+1}\right\| \leq \widetilde{H}\left(W\left(y_{n}\right), W\left(y_{n+1}\right)\right), \\
x_{n+1}=\left(1-\alpha_{n}\right) x_{n}+\alpha_{n} J_{A}^{M}\left[g\left(y_{n}\right)-\rho T\left(v_{n}, u_{n}\right)\right] \text { for } \rho>0, \\
y_{n}=\left(1-\beta_{n}\right) x_{n}+\beta_{n} J_{A}^{M}\left[g\left(x_{n}\right)-\eta T\left(u_{n}, v_{n}\right)\right] \text { for } \eta>0,
\end{gathered}
$$

where $\left\{\alpha_{n}\right\}$ and $\left\{\beta_{n}\right\}$ are sequences in $[0,1]$.

If $T: E \rightarrow E$ is a univariate mapping and $\beta_{n}=1$, then the Algorithm 2.1 reduces to the following.

Algorithm 2.2. For given $x_{0}, y_{0} \in E, u_{0} \in V\left(x_{0}\right), v_{0} \in W\left(y_{0}\right)$, compute the sequences $\left\{x_{n}\right\},\left\{y_{n}\right\},\left\{u_{n}\right\}$ and $\left\{v_{n}\right\}$ from the iterative schemes

$$
\begin{gathered}
u_{n} \in V\left(x_{n}\right):\left\|u_{n}-u_{n+1}\right\| \leq \widetilde{H}\left(V\left(x_{n}\right), V\left(x_{n+1}\right)\right), \\
v_{n} \in W\left(y_{n}\right):\left\|v_{n}-v_{n+1}\right\| \leq \widetilde{H}\left(W\left(y_{n}\right), W\left(y_{n+1}\right)\right), \\
x_{n+1}=\left(1-\alpha_{n}\right) x_{n}+\alpha_{n} J_{A}^{M}\left[g\left(y_{n}\right)-\rho T\left(v_{n}\right)\right] \text { for } \rho>0, \\
y_{n}=J_{A}^{M}\left[g\left(x_{n}\right)-\eta T\left(u_{n}\right)\right] \text { for } \eta>0,
\end{gathered}
$$

where $\alpha_{n} \in[0,1], \forall n \geq 0$.

Remark 2.1. If $E=H$ is a Hilbert space, $M=g=I$ and $A=\partial \phi$, where $\phi$ is the indicator function of a closed convex subset $K$ in $H$, then Algorithm 2.1 reduces to the Algorithm 2.1 in [2].

In order to prove our main results we need the following lemma:

Lemma $2.3([2])$. Let $\left\{a_{n}\right\},\left\{b_{n}\right\}$ and $\left\{c_{n}\right\}$ be three nonnegative real sequences satisfying the following conditions:

$$
a_{n+1} \leq\left(1-\lambda_{n}\right) a_{n}+b_{n}+c_{n}, \quad \forall n \geq n_{0},
$$

where $n_{0}$ is some nonnegative integer, $\lambda_{n} \in(0,1)$ with $\sum_{n=0}^{\infty} \lambda_{n}=\infty, b_{n}=$ $o\left(\lambda_{n}\right)$ and $\sum_{n=0}^{\infty} c_{n}<\infty$, then $a_{n} \rightarrow 0($ as $n \rightarrow \infty)$.

We now present, based on Algorithm 2.1, the approximation-solvability of the SNISVI problem (1) and (2) involving a mapping $T: E \times E \rightarrow E$ which is relaxed $(\gamma, r)$-cocoercive with respect to $(V, g)$ and $(W, g)$ in the first variable, respectively.

Theorem 2.4. Let $E$ be a real q-uniformly smooth Banach space, $M: E \rightarrow E$ be a strongly accretive operator with constant $k$ and $A: E \rightarrow 2^{E}$ be a $M$ accretive operator. Let $g: E \rightarrow E$ be $\sigma$-Lipschitz continuous. Assume that $V, W: E \rightarrow C(E)$ be $\widetilde{H}$-Lipschitz continuous with constant $\beta>0$ and $\xi>0$, respectively. Let $T: E \times E \rightarrow E$ be relaxed $(\gamma, r)$-cocoercive with respect to $(V, g)$ and $(W, g)$ in the first variable, respectively. And let $T: E \times E \rightarrow E$ 
be $\mu$-Lipschitz continuous in the first variable. Suppose that $\left(x^{*}, y^{*}, u^{*}, v^{*}\right) \in$ $E \times E \times E \times E$ is a solution to the SNISVI problem (1) and (2) and that $\left\{x_{n}\right\}$, $\left\{y_{n}\right\},\left\{u_{n}\right\}$, and $\left\{v_{n}\right\}$ are the sequences generated by Algorithm 2.1. If the following conditions are satisfied:

(i) $\sum_{n=0}^{\infty} \alpha_{n}=\infty$;

(ii) $\sum_{n=0}^{\infty}\left(1-\beta_{n}\right)<\infty$;

(iii) $0<\rho<\sqrt[q-1]{\frac{q\left(r-\gamma \mu^{q} \xi^{q}\right)}{c_{q} \mu^{q} \xi^{q}}}, \quad 0<\eta<\sqrt[q-1]{\frac{q\left(r-\gamma \mu^{q} \beta^{q}\right)}{c_{q} \mu^{q} \beta^{q}}}$;

(iv) $r>\max \left\{\gamma \mu^{q} \xi^{q}, \gamma \mu^{q} \beta^{q}\right\}, \quad \sigma \leq k$,

then the sequence $\left\{x_{n}\right\},\left\{y_{n}\right\},\left\{u_{n}\right\}$ and $\left\{v_{n}\right\}$ converges strongly to $x^{*}, y^{*}, u^{*}$ and $v^{*}$ in E, respectively.

Proof. Since $\left(x^{*}, y^{*}, u^{*}, v^{*}\right)$ is a solution to the SNISVI problem (1) and (2), it follows from Lemma 2.2 that

$$
x^{*}=J_{A}^{M}\left[g\left(y^{*}\right)-\rho T\left(v^{*}, u^{*}\right)\right], \quad y^{*}=J_{A}^{M}\left[g\left(x^{*}\right)-\eta T\left(u^{*}, v^{*}\right)\right] .
$$

It follows from Algorithm 2.1 and Lemma 1.5 that

$$
\begin{aligned}
\left\|x_{n+1}-x^{*}\right\|= & \|\left(1-\alpha_{n}\right) x_{n}+\alpha_{n} J_{A}^{M}\left[g\left(y_{n}\right)-\rho T\left(v_{n}, u_{n}\right)\right] \\
& \quad-\left(1-\alpha_{n}\right) x^{*}-\alpha_{n} J_{A}^{M}\left[g\left(y^{*}\right)-\rho T\left(v^{*}, u^{*}\right)\right] \| \\
\leq & \left(1-\alpha_{n}\right)\left\|x_{n}-x^{*}\right\|+\alpha_{n} \| J_{A}^{M}\left[g\left(y_{n}\right)-\rho T\left(v_{n}, u_{n}\right)\right] \\
& \quad-J_{A}^{M}\left[g\left(y^{*}\right)-\rho T\left(v^{*}, u^{*}\right)\right] \| \\
\leq & \left(1-\alpha_{n}\right)\left\|x_{n}-x^{*}\right\| \\
& \quad+\frac{\alpha_{n}}{k}\left\|g\left(y_{n}\right)-g\left(y^{*}\right)-\rho\left(T\left(v_{n}, u_{n}\right)-T\left(v^{*}, u^{*}\right)\right)\right\| .
\end{aligned}
$$

Since $T$ is relaxed $(\gamma, r)$-cocoercive with respect to $(W, g)$ and $\mu$-Lipschitz continuous in the first variable, respectively, we have by Lemma 2.1 and the $\xi-\widetilde{H}$-Lipschitz continuity of $W$ that

$$
\begin{aligned}
& \left\|g\left(y_{n}\right)-g\left(y^{*}\right)-\rho\left(T\left(v_{n}, u_{n}\right)-T\left(v^{*}, u^{*}\right)\right)\right\|^{q} \\
\leq & \left\|g\left(y_{n}\right)-g\left(y^{*}\right)\right\|^{q}-\rho q\left\langle T\left(v_{n}, u_{n}\right)-T\left(v^{*}, u^{*}\right), J_{q}\left(g\left(y_{n}\right)-g\left(y^{*}\right)\right)\right\rangle \\
& \quad+c_{q} \rho^{q}\left\|T\left(v_{n}, u_{n}\right)-T\left(v^{*}, u^{*}\right)\right\|^{q} \\
\leq & \sigma^{q}\left\|y_{n}-y^{*}\right\|^{q}+c_{q} \rho^{q} \mu^{q}\left\|v_{n}-v^{*}\right\|^{q}+\rho q \gamma\left\|T\left(v_{n}, u_{n}\right)-T\left(v^{*}, u^{*}\right)\right\|^{q} \\
& \quad-q \rho r\left\|y_{n}-y^{*}\right\|^{q} \\
\leq & \sigma^{q}\left\|y_{n}-y^{*}\right\|^{q}+c_{q} \rho^{q} \mu^{q} \xi^{q}\left\|y_{n}-y^{*}\right\|^{q} \\
& \quad+\rho q \gamma \mu^{q}\left\|v_{n}-v^{*}\right\|^{q}-q \rho r\left\|y_{n}-y^{*}\right\|^{q} \\
\leq & \sigma^{q}\left[1+\frac{c_{q} \rho^{q} \mu^{q} \xi^{q}+\rho q \gamma \mu^{q} \xi^{q}-q \rho r}{\sigma^{q}}\right]\left\|y_{n}-y^{*}\right\|^{q} .
\end{aligned}
$$


Substituting (12) into (11), we get by conditions (iii) and (iv) that

$$
\begin{aligned}
\left\|x_{n+1}-x^{*}\right\| & \leq\left(1-\alpha_{n}\right)\left\|x_{n}-x^{*}\right\|+\frac{\sigma}{k} \alpha_{n} \Omega_{1}\left\|y_{n}-y^{*}\right\| \\
& \leq\left(1-\alpha_{n}\right)\left\|x_{n}-x^{*}\right\|+\alpha_{n} \Omega_{1}\left\|y_{n}-y^{*}\right\|
\end{aligned}
$$

where $\Omega_{1}=\sqrt[q]{1+\frac{c_{q} \rho^{q} \mu^{q} \xi^{q}+\rho q \gamma \mu^{q} \xi^{q}-q \rho r}{\sigma^{q}}}<1$ (By condition (iii)).

Next, we make an estimation for $\left\|y_{n}-y^{*}\right\|$. Again applying Algorithm 2.1 and Lemma 1.5, we have

(14)

$$
\begin{aligned}
\left\|y_{n}-y^{*}\right\|= & \|\left(1-\beta_{n}\right) x_{n}+\beta_{n} J_{A}^{M}\left[g\left(x_{n}\right)-\eta T\left(u_{n}, v_{n}\right)\right] \\
& \quad-\left(1-\beta_{n}\right) y^{*}-\beta_{n} J_{A}^{M}\left[g\left(x^{*}\right)-\eta T\left(u^{*}, v^{*}\right)\right] \| \\
\leq & \left(1-\beta_{n}\right)\left\|x_{n}-y^{*}\right\|+\frac{\beta_{n}}{k}\left\|g\left(x_{n}\right)-g\left(x^{*}\right)-\eta\left(T\left(u_{n}, v_{n}\right)-T\left(u^{*}, v^{*}\right)\right)\right\| \\
\leq & \left(1-\beta_{n}\right)\left\|x_{n}-x^{*}\right\|+\left(1-\beta_{n}\right)\left\|x^{*}-y^{*}\right\| \\
& \quad+\frac{\beta_{n}}{k}\left\|g\left(x_{n}\right)-g\left(x^{*}\right)-\eta\left(T\left(u_{n}, v_{n}\right)-T\left(u^{*}, v^{*}\right)\right)\right\| .
\end{aligned}
$$

Since $T$ is relaxed $(\gamma, r)$-cocoercive with respect to $(V, g)$ and $\mu$-Lipschitz continuous in the first variable respectively, and $V$ is $\beta-\widetilde{H}$-Lipschitz continuous, similar to the proof of (12), we obtain that

$$
\begin{aligned}
& \left\|g\left(x_{n}\right)-g\left(x^{*}\right)-\eta\left(T\left(u_{n}, v_{n}\right)-T\left(u^{*}, v^{*}\right)\right)\right\|^{q} \\
\leq & \sigma^{q}\left[1+\frac{c_{q} \eta^{q} \mu^{q} \beta^{q}+\eta q \gamma \mu^{q} \beta^{q}-q \eta r}{\sigma^{q}}\right]\left\|x_{n}-x^{*}\right\|^{q} .
\end{aligned}
$$

Combining (14), (15) and condition (iv), we have

$$
\begin{aligned}
\left\|y_{n}-y^{*}\right\| & \leq\left(1-\beta_{n}\right)\left\|x_{n}-x^{*}\right\|+\left(1-\beta_{n}\right)\left\|x^{*}-y^{*}\right\|+\frac{\sigma}{k} \beta_{n} \Omega_{2}\left\|x_{n}-x^{*}\right\| \\
& \leq\left(1-\beta_{n}\right)\left\|x_{n}-x^{*}\right\|+\left(1-\beta_{n}\right)\left\|x^{*}-y^{*}\right\|+\beta_{n} \Omega_{2}\left\|x_{n}-x^{*}\right\| \\
& \leq\left\|x_{n}-x^{*}\right\|+\left(1-\beta_{n}\right)\left\|x^{*}-y^{*}\right\|,
\end{aligned}
$$

where $\Omega_{2}=\sqrt[q]{1+\frac{c_{q} \eta^{q} \mu^{q} \beta^{q}+\eta q \gamma \mu^{q} \beta^{q}-q \eta r}{\sigma^{q}}}<1$ (By condition (iii)).

Substituting (16) into (13), we get

$$
\begin{aligned}
\left\|x_{n+1}-x^{*}\right\| & \leq\left(1-\alpha_{n}\right)\left\|x_{n}-x^{*}\right\|+\alpha_{n} \Omega_{1}\left[\left\|x_{n}-x^{*}\right\|+\left(1-\beta_{n}\right)\left\|x^{*}-y^{*}\right\|\right] \\
& \leq\left(1-\alpha_{n}\left(1-\Omega_{1}\right)\right)\left\|x_{n}-x^{*}\right\|+\Omega_{1}\left(1-\beta_{n}\right)\left\|x^{*}-y^{*}\right\| .
\end{aligned}
$$

Taking $a_{n}=\left\|x_{n}-x^{*}\right\|, \lambda_{n}=\alpha_{n}\left(1-\Omega_{1}\right), b_{n}=0$ and $c_{n}=\Omega_{1}\left(1-\beta_{n}\right)\left\|x_{*}-y^{*}\right\|$ in Lemma 2.3, we know that all conditions in Lemma 2.3 are satisfied, and so $x_{n} \rightarrow x^{*} \in E($ as $n \rightarrow \infty)$. Since $V$ is $\beta$-Lipschitz continuous, we obtain

$$
\left\|u_{n}-u^{*}\right\| \leq \widetilde{H}\left(V\left(x_{n}\right), V\left(x^{*}\right)\right) \leq \beta\left\|x_{n}-x^{*}\right\| \rightarrow 0, \quad n \rightarrow \infty,
$$

i.e., $u_{n} \rightarrow u^{*} \in E($ as $n \rightarrow \infty)$. Similarly, we have that $v_{n} \rightarrow v^{*}, n \rightarrow \infty$. 
We now show that $u^{*} \in V\left(x^{*}\right)$ and $v^{*} \in W\left(y^{*}\right)$. In fact,

$$
\begin{aligned}
d\left(u^{*}, V\left(x^{*}\right)\right) & \leq\left\|u^{*}-u_{n}\right\|+d\left(u_{n}, V\left(x^{*}\right)\right) \\
& \leq\left\|u^{*}-u_{n}\right\|+\widetilde{H}\left(V\left(x_{n}\right), V\left(x^{*}\right)\right) \\
& \leq\left\|u^{*}-u_{n}\right\|+\beta\left\|x_{n}-x^{*}\right\| \rightarrow 0 \quad \text { as } n \rightarrow \infty
\end{aligned}
$$

where $d\left(u^{*}, V\left(x^{*}\right)\right)=\inf \left\{\left\|u^{*}-z\right\|: z \in V\left(x^{*}\right)\right\}$. This implies that $u^{*} \in V\left(x^{*}\right)$. In a similar way, one show that $v^{*} \in W\left(y^{*}\right)$. This completes the proof.

Remark 2.2. If $E=H$ is a Hilbert space, $M=g=I$ and $A=\partial \phi$, where $\phi$ is the indicator function of a closed convex subset $K$ in $H$, then $q=2, c_{2}=1$ (by [10]), $\sigma=k=\xi=\beta=1$, and Theorem 2.4 reduces to Theorem 3.1 in [2]. Obviously, Theorem 2.4 extends and improves the main results in Verma $[8,9]$.

Acknowledgements. The author would like to thank the referees for their valuable suggestions and comments. Project supported by the National Natural Science Foundation of China (10771212) and the Natural Science Foundation of Jiangsu Education Office (06KJB110010).

\section{References}

[1] C. Z. Bai and J. X. Fang, A system of nonlinear variational inclusions in real Banach spaces, Bull. Korean Math. Soc. 40 (2003), no. 3, 385-397.

[2] S. S. Chang, H. W. Joseph Lee, and C. K. Chan, Generalized system for relaxed cocoercive variational inequalities in Hilbert spaces, Appl. Math. Lett. 20 (2007), no. 3, 329-334.

[3] K. Deimling, Nonlinear Functional Analysis, Springer-Verlag, Berlin, 1985.

[4] Y. P. Fang and N. J. Huang, H-accretive operators and resolvent operator technique for solving variational inclusions in Banach spaces, Appl. Math. Lett. 17 (2004), no. 6, 647-653.

[5] _ H-Monotone operator and resolvent operator technique for variational inclusions, Appl. Math. Comput. 145 (2003), no. 2-3, 795-803.

[6] H. Nie, Z. Liu, K. H. Kim, and S. M. Kang, A system of nonlinear variational inequalities involving strongly monotone and pseudocontractive mappings, Adv. Nonlinear Var. Inequal. 6 (2003), no. 2, 91-99.

[7] M. Aslam Noor, Three-step iterative algorithms for multivalued quasi variational inclusions, J. Math. Anal. Appl. 255 (2001), no. 2, 589-604.

[8] R. U. Verma, Generalized system for relaxed cocoercive variational inequalities and its projection methods, J. Optim. Theory Appl. 121 (2004), no. 1, 203-210.

[9] __ General convergence analysis for two-step projection methods and applications to variational problem, Appl. Math. Lett. 18 (2005), no. 11, 1286-1292.

[10] H. K. Xu, Inequalities in Banach spaces with applications, Nonlinear Anal. 16 (1991), no. $12,1127-1985$. 


\section{Chuanzhi Bai}

Department of Mathematics

HuAiYin Normal University

Huaian, Jiangsu 223300, P. R. China

E-mail address: czbai8@sohu.com

QING YANG

Department of Mathematics

Huaiyin Normal University

Huaian, Jiangsi 223001, P. R. China

E-mail address: yangqing3511115@163.com 